\title{
The Effect of Hounsfield Unit Value with Conventional Computed Tomography and Intraoperative Distraction on Postoperative Intervertebral Height Reduction in Patients Following Stand-Alone Anterior Cervical Discectomy and Fusion
}

\author{
Jun Seok Lee, ${ }^{1,2,3}$ Dong Wuk Son, ${ }^{1,2,3}$ Su Hun Lee, ${ }^{1,2,3}$ Sung Soon Ki, ${ }^{1,2,3}$ Sang Weon Lee, ${ }^{1,2,3}$ Geun Sung Song, ${ }^{1,2,3}$ \\ Joon Bum Woo, ${ }^{1,2,3}$ Young Ha Kim ${ }^{1,2,3}$ \\ Department of Neurosurgery, Pusan National University Yangsan Hospital, Yangsan, Korea \\ Research Institute for Convergence of Biomedical Science and Technology, ${ }^{2}$ Pusan National University Yangsan Hospital, Yangsan, Korea \\ Department of Neurosurgery, ${ }^{3}$ School of Medicine, Pusan National University, Yangsan, Korea
}

\begin{abstract}
Objective : The most common complication of anterior cervical discectomy and fusion (ACDF) is cage subsidence and maintenance of disc height affects postoperative clinical outcomes. We considered cage subsidence as an inappropriate indicator for evaluating preservation of disc height. Thus, this study aimed to consider patients with complications such as reduced total disc height compared to that before surgery and evaluate the relevance of several factors before ACDF.

Methods : We retrospectively reviewed the medical records of 40 patients who underwent stand-alone single-level ACDF using a polyetheretherketone (PEEK) cage at our institution between January 2012 and December 2018. Our study population comprised 19 male and 21 female patients aged 24-70 years. The minimum follow-up period was 1 year. Twenty-seven patients had preoperative bone mineral density (BMD) data on dual-energy X-ray absorptiometry. Clinical parameters included sex, age, body mass index, smoking history, and prior medical history. Radiologic parameters included the C2-7 cobb angle, segmental angle, sagittal vertical axis, disc height, and total intervertebral height $(\mathrm{TIH})$ at the preoperative and postoperative periods. Cage decrement was defined as the reduction in $\mathrm{TIH}$ at the 6 -month follow-up compared to preoperative TIH. To evaluate the bone quality, Hounsfield unit (HU) value was calculated in the axial and sagittal images of conventional computed tomography.
\end{abstract}

Results : Lumbar BMD values and cervical HU values were significantly correlated $(r=0.733, p<0.001)$. We divided the patients into two groups based on cage decrement, and $47.5 \%$ of the total patients were regarded as cage decrement. There were statistically significant differences in the parameters of measuring the $\mathrm{HU}$ value of the vertebra and intraoperative distraction between the two groups. Using these identified factors, we performed a receiver operating characteristic (ROC) curve analysis. Based on the ROC curve, the cut-off point was 530 at the $\mathrm{HU}$ value of the upper cortical and cancellous vertebrae ( $p=0.014$; area under the curve [AUC], 0.727 ; sensitivity, $94.7 \%$; specificity, $42.9 \%$ ) and 22.41 at intraoperative distraction ( $p=0.017$; AUC, 0.722 ; sensitivity, $85.7 \%$; specificity, $57.9 \%)$. Using this value, we converted these parameters into a bifurcated variable and assessed the multinomial regression analysis to evaluate the risk factors for cage decrement in ACDF. Intraoperative distraction and HU value of the upper vertebral body were independent factors of postoperative subsidence.

Conclusion : Insufficient intraoperative distraction and low HU value showed a strong relationship with postoperative intervertebral height reduction following single stand-alone PEEK cage ACDF.

Key Words : Cervical vertebrae · Prosthesis failure · Risk assessment · Osteoporosis · Computed tomography.

- Received : May 26, 2021 •Revised : July 16, 2021 •Accepted : August 4, 2021

- Address for reprints : Dong Wuk Son

Department of Neurosurgery, Pusan National University Yangsan Hospital, 20 Geumo-ro, Mulgeum-eup, Yangsan 50612, Korea

Tel : +82-55-360-2126, Fax : +82-55-360-2156, E-mail : md6576@naver.com, ORCID : https://orcid.org/0000-0002-9154-1923

This is an Open Access article distributed under the terms of the Creative Commons Attribution Non-Commercial License (http://creativecommons.org/licenses/by-nc/4.0) which permits unrestricted non-commercial use, distribution, and reproduction in any medium, provided the original work is properly cited. 


\section{INTRODUCTION}

Anterior cervical discectomy and fusion (ACDF) is a common surgical method for treating patients with degenerative cervical spinal diseases. This procedure could effectively remove compressive lesions located in front of the cervical medulla or cervical nerve roots, maintain segmental lordosis, and recover the intervertebral disc height. Initially, an autologous bone graft from the iliac crest was used as a substitute after removal of the intervertebral disc; however, several alternative devices such as polyetheretherketone (PEEK) cages, carbon-fiber cages, and three-dimensional printed titanium cages have been developed to reduce donor site morbidity ${ }^{8,16)}$. Among them, PEEK cages have been widely used to replace autologous bone grafts ${ }^{10)}$.

ACDF with stand-alone PEEK cage (SA PEEK cage) had some complications, such as pseudoarthrosis, graft expulsion or collapse, cage migration, and cage subsidence. Restoring disc height is important for patients undergoing ACDF surgery, and cage subsidence is the major complication that reduces disc height ${ }^{21)}$. There is no consensus on the definition of cage subsidence. However, a disc height reduction of $\geq 2 \mathrm{~mm}$ or $\geq 3 \mathrm{~mm}$ between immediately after surgery and the last follow-up was defined as cage subsidence ${ }^{21)}$.

To date, several studies have reported the risk factors affecting postoperative cage subsidence after ACDF with SA PEEK cages. The risk factor for cage subsidence is the distance of the cage from the anterior vertebral rim, preoperative cervical kyphosis, cage location, over-distraction, and excessive endplate preparation $^{1,20)}$.

The traditional concept of cage subsidence is an inappropriate indicator for evaluating the preservation of disc height and prognosis of ACDF surgery. This is because, first, cage subsidence was theoretically expected to decrease the intervertebral neural foramen and worsen radicular pain. However, it is uncertain whether cage subsidence after ACDF affects the clinical prognosis ${ }^{10,20)}$. Cage subsidence was only evaluated based on the amount of cage deposited into the vertebral body. Cage subsidence is difficult to verify if the postoperative disc height is maintained compared to that before surgery. Although postoperative cage subsidence occurs, if there is enough intraoperative distraction during surgery, intervertebral foraminal height can be maintained and symptoms related to spinal stenosis can be mitigated by sufficient distraction. Therefore, based on the changes in intervertebral disc height rather than cage subsidence, we planned the study to consider patients with complications such as reduced total disc height compared to that before surgery.

Second, few studies on the risk factors of subsidence have been conducted in patients with osteoporosis. Several studies have been conducted to confirm the relationship between osteoporosis and cage subsidence; however, no significant correlation has been identified.

Dual-energy absorptiometry (DEXA) is a common diagnostic tool for osteoporosis ${ }^{4}$. However, DEXA often fails to reflect the severe degenerative spine of the elderly ${ }^{6}$. Additionally, in cervical spine surgery, the bone condition of the cervical spine cannot be measured directly using DEXA. Quantitative computed tomography (CT) is currently the only method that can directly measure the BMD of the cervical spine ${ }^{12)}$. However, the maintenance cost of the equipment is expensive and is not widely used, making it inappropriate as a standard test method. We considered a way to effectively assess cervical bone quality without introducing new equipment preoperatively. The region of interest (ROI) value of measuring the Hounsfield unit (HU) in conventional CT could reflect the quality of the cervical bone to some extent.

In this study, we first evaluated whether the ROI values of conventional cervical CT are correlated with the lumbar DEXA BMD; if the ROI value of cervical bone was meaningful, and after classifying the cage decrement with the new criteria aforementioned, we evaluated the relationship with postoperative disc height decrement after stand-alone ACDF surgery.

\section{MATERIALS AND METHODS}

\section{Patient demographics}

The study protocol was approved by the Institutional Review Board of Pusan National University Yangsan Hospital, which waived the requirement for informed consent due to the retrospective nature of this study (IRB No. 05-2021-115). We retrospectively reviewed the medical records of 40 patients who underwent single-level ACDF using a stand-alone PEEK cage between January 2012 and November 2018. All patients were treated for single-level pathology of the sub-axial cervical level by an experienced surgeon. The mean age was 52 years 
(range, 24-73). The inclusion criteria were as follows : 1) patients with neck pain and radiculopathy lasting for 3 months based on concordant preoperative magnetic resonance imaging (MRI); 2) cervical myelopathy due to acute rupture of the cervical disc; 3) all patients underwent a minimum 1-year follow-up period postoperatively with proper radiological examinations performed in outpatient clinics, and 4) only cases showing C7 and T1 vertebral body in the preoperative cervical sagittal plane examination.

\section{Surgical procedure}

All patients underwent a standard Smith-Robinson anterior approach to the cervical spine. After adequate exposure of the operative lesions, a Casper cervical retractor was placed, and a discectomy was performed in the standard manner. For complete decompression, we removed the bilateral subtotal uncinate process, and osteophytes of the posterior part of the vertebral body with high speed electric drill and Kerrison punch. We performed the bilateral partial uncinectomy to remove the remnant osteophyte regrowth, even in patients with unilateral symptoms. And we proceeded the uncinectomy laterally until the nerve root was fully decompressed. Posterior longitudinal ligament was selected removed for the patients in which ruptured disc directly compressed the dura. After decompression, the adjacent cartilaginous endplate was carefully removed to avoid damage to the bony endplate. And we found the proper cage size by inserting a different sized trial cages. Appropriate sized was selected according to immobility of trial cages following distractor removal. The implant was inserted under intraoperative fluoroscopy guidance. In all cases, a standalone anchored PEEK cage (Cornerstone-SR ${ }^{\circledR}$; Medtronic Sofamor Danek, Memphis, TN, USA) was inserted without anterior plate anchoring. All patients were instructed to wear a soft collar for 2 months, postoperatively.

\section{Radiologic assessment}

All radiologic assessments were performed by two independent neurological patients who were not involved in the study and blinded to all clinical information. Routine preoperative radiological examinations consisted of plain radiographs (standing anteroposterior, lateral neutral, lateral flexion, lateral extension, and bilateral oblique views), CT, and MRI.

Radiologic parameters on plain radiographs included the C2-7 cobb angle (CA), T1 slope, C2-7 sagittal vertical axis
(SVA), range of motion from C2-7, segmental angle (SA), total intervertebral height (TIH), and disc height. The C2-7 SVA, T1 slope, C2-7 CA, cage location, and intraoperative distrac-

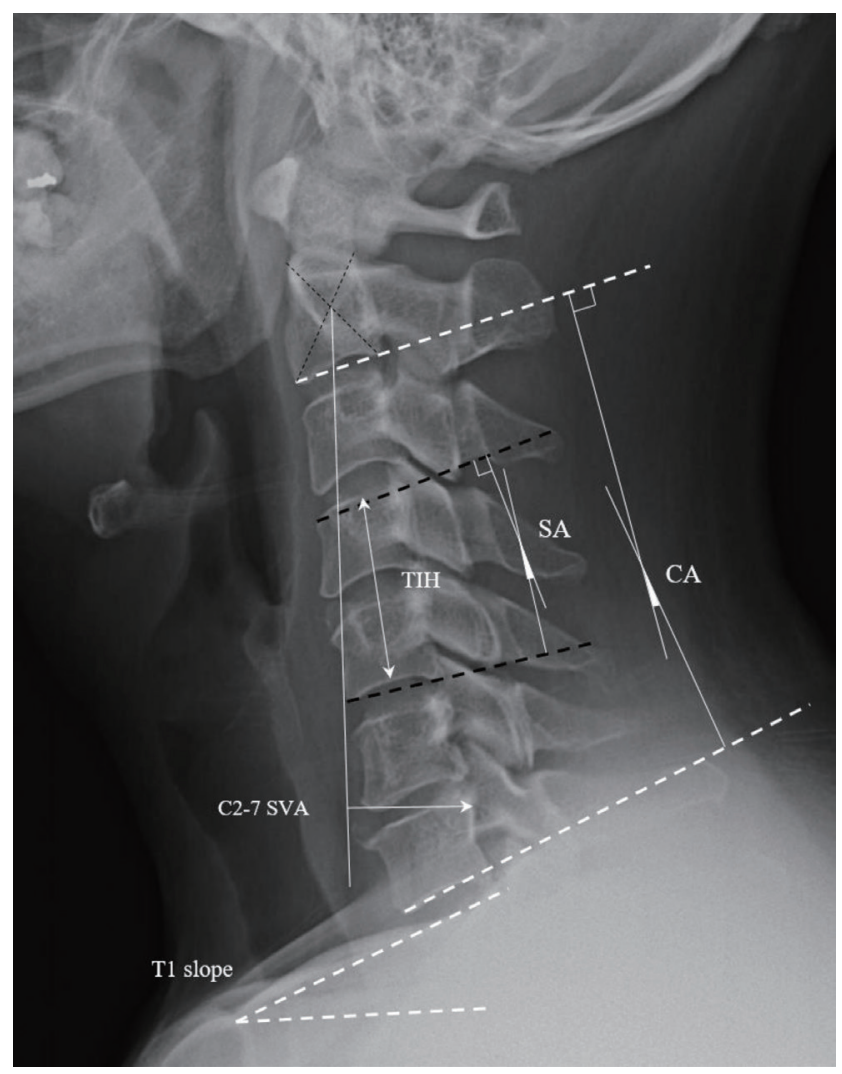

Fig. 1. Measurement of the preoperative radiologic parameters. $\mathrm{TIH}$ : total intervertebral height, SA : segmental angle, CA : C2-7 cobb angle, SVA : sagittal vertical axis.
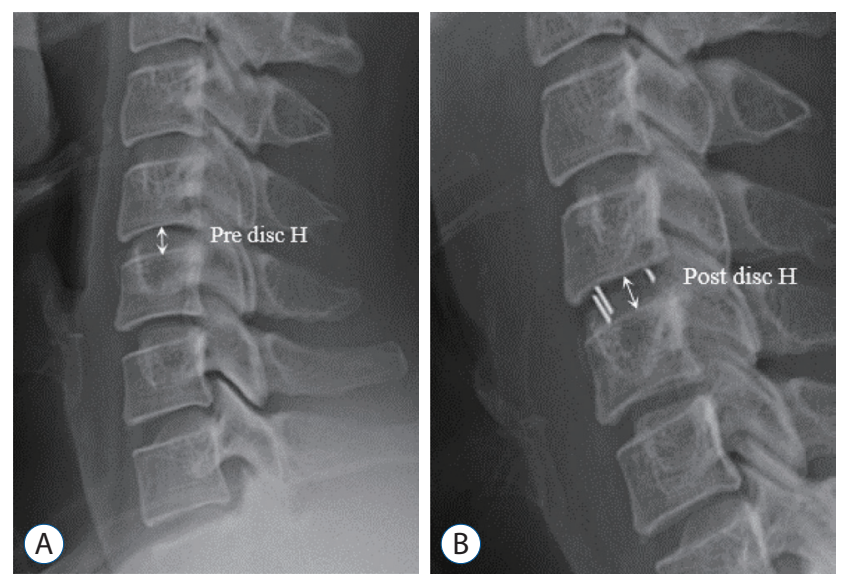

Fig. 2. $A$ and $B$ : Definition of intraoperative distraction, [(post disc $\mathrm{H}$ pre disc $\mathrm{H}$ ) / pre disc $\mathrm{H}] \times 100.0(\%)]$. $\mathrm{H}$ : height ant mid portion of disc space. 
tion were assessed on cervical neutral lateral radiographs in the free-standing position, with the patient's head maintaining a horizontal gaze. Plain images were taken preoperatively and at postoperative day (POD) 1 and at 6 months, and 1 year postoperatively. TIH was defined as the distance from the midpoint of the upper endplate of the cephalic cervical vertebra to the lower endplate of the caudal cervical vertebra. Intraoperative distraction was assessed as the ratio of preoperative disc height to incremented disc height postoperatively. Cage decrement was defined as the reduction in TIH at the 6-month follow-up compared to preoperative TIH. Intraoperative distraction was measured as per the study by Barsa and Suchomel ${ }^{1)}$, and pseudoarthrosis was defined as segmental instability with a $2 \mathrm{~mm}$ alteration of the inter-spinous distance or a $\geq 2^{\circ}$ increase in the $\mathrm{SA}^{21}$. All radiologic parameters were measured using commercial software (Marosis 5.0; INFINITT Healthcare, Seoul, Korea) and these measurement methods are presented in Figs. 1 and 2.

To assess the attenuation of the vertebral body, high-resolution CT scans of the cervical spine were retrospectively analyzed using a commercially available picture archiving and communication system (Somatom FORCE; Siemens, Forchheim, Germany). Two-dimensional reconstruction images were acquired in the axial and sagittal planes. CT attenuation was measured in HU by placing a click-and-drag elliptical ROI using a sagittal and axial CT image. HU values were calculated at the vertebra above and below the bone graft placement. In the sagittal image, HU values were measured in the lower half of the upper vertebra and the upper half of the lower vertebra at the midline. In the axial image, the $\mathrm{HU}$ value was measured in the mid-portion of the upper and lower vertebrae. To evaluate the effect of cortical bone thickness, HU values were measured by distinguishing the areas that con- tained the cortical bone. The measurement methods are shown in Fig. 3.

\section{Statistical analysis}

Statistical analysis was performed using SPSS (version 24.0; SPSS Inc., Chicago, IL, USA). Statistical significance was set at $p<0.05$. Normally distributed data of the groups were evaluated using the Student's t-test and Mann-Whitney U test for parametric and nonparametric continuous variables, respectively. The Pearson correlation coefficient analysis was performed to confirm the relationship between continuous variables (radiologic parameters). A multiple logistic regression analysis was performed to determine the risk factors for subsidence. A receiver operating characteristic (ROC) curve analysis was used to determine the cut-off value, which was defined as the point corresponding to the maximum sum of the sensitivity and specificity.

\section{RESULTS}

The medical records and radiologic examinations of $40 \mathrm{pa}-$ tients were retrospectively analyzed. Our study included 19 male and 21 female patients aged between 24 and 73 years. The mean age of the patients was 52 years. Minimum followup period of all patients was 1 year and mean follow-up period was 18 months. Postoperative radiologic plain examination was taken at 1 day and at 6 months, and 1 year postoperatively.

For patients who underwent DEXA examination preoperatively, we analyzed the relationship between BMD values measured using DEXA and calculated $\mathrm{HU}$ values. The $\mathrm{HU}$ value showed a higher correlation with lumbar DEXA scores and a weak correlation with the T-score. However, it did not corre-
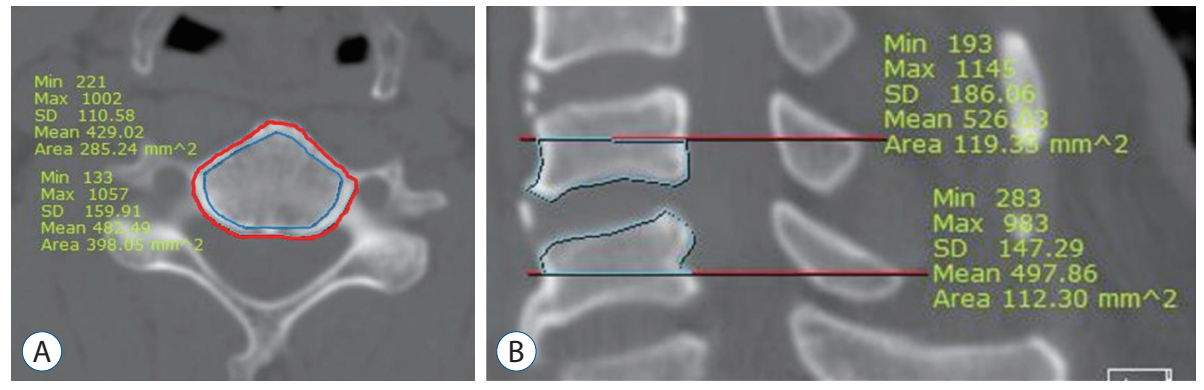

Fig. 3. Measurement of Hounsfield units. A : Axial image of preoperative computed tomography (CT) image. Red line : Region of interest (ROI) area including cortical bone and cancellous bone, blue line : ROI area including cancellous bone. B : Sagittal section of preoperative CT image. SD : standard deviation. 
late with the femur BMD score. The HU value did not differ significantly depending on whether the cortical bone was included, and the HU value measured in the upper cortical bone was the most significant with DEXA BMD values. The results are summarized in Table 1.

Since the association of HU values and BMD has been verified, we used the $\mathrm{HU}$ values to assess the relevance of the subsidence.

Approximately $47.5 \%$ of the total patients were regarded as cage decrements. Cage decrement mostly occurred at the C5/6 level. However, there was no statistical relevance according to the surgical level, and no patient underwent revision surgery due to postoperative complications. We analyzed the clinical characteristics of patients with postoperative cage decrement. The demographic characteristics of the two groups are summarized in Table 2. No significant differences were found among clinical parameters. There were no differences in the preoperative radiologic parameters between the two groups.

In Table 3, we presented the radiologic change of the two groups. Regarding radiologic parameters, the cervical angle was almost unchanged, and there was no difference between the two groups. However, there were significant differences in the parameters related to intraoperative changes, such as variations in SA, TIH, and intraoperative distractions. There were also statistically significant differences in the parameters for measuring the $\mathrm{HU}$ value of the vertebral body at the surgery site. The HU value including cortical bone was approximately 70 units higher than that of the group that calculated only the cancellous bone. However, there was no difference in the results, even if the cortical bone was included. Among the areas for which the HU value was measured, the measurement of the upper vertebral body including cortical bone was the most significant area $(p=0.011)$.

Using these identified factors, we performed an ROC curve analysis. Sensitivity and specificity were calculated for each cut-off value and the area under the curve (Table 4). The cutoff point was specified from the ROC curve using the optimal intersection of specificity and sensitivity. Based on the ROC curve, the cut-off point was $530 \mathrm{HU}$ at the upper cortical and cancellous vertebrae $(p=0.014$; area under the curve [AUC],

Table 1. Results of Pearson correlations between region of HU value and DEXA

\begin{tabular}{|c|c|c|c|c|}
\hline \multirow{2}{*}{ Region of HU value } & \multicolumn{2}{|c|}{ Lumbar BMD } & \multicolumn{2}{|c|}{ Femur BMD } \\
\hline & Area $\left(\mathrm{g} / \mathrm{cm}^{2}\right)$ & T-score & Area $\left(\mathrm{g} / \mathrm{cm}^{2}\right)$ & T-score \\
\hline \multicolumn{5}{|c|}{ Upper vertebra cancellous } \\
\hline r & 0.685 & 0.733 & 0.367 & 0.525 \\
\hline$p$-value & $<0.001$ & $<0.001$ & 0.065 & 0.002 \\
\hline \multicolumn{5}{|c|}{ Upper vertebra cortical + cancellous } \\
\hline r & 0.683 & 0.730 & 0.258 & 0.505 \\
\hline$p$-value & $<0.001$ & $<0.001$ & 0.204 & 0.009 \\
\hline \multicolumn{5}{|c|}{ Lower vertebra cancellous } \\
\hline r & 0.662 & 0.738 & 0.318 & 0.617 \\
\hline$p$-value & $<0.001$ & $<0.001$ & 0.114 & 0.001 \\
\hline \multicolumn{5}{|c|}{ Lower vertebra cortical + cancellous } \\
\hline r & 0.544 & 0.634 & 0.178 & 0.519 \\
\hline$p$-value & 0.003 & $<0.001$ & 0.385 & 0.007 \\
\hline \multicolumn{5}{|l|}{ Upper vertebra, sagittal } \\
\hline r & 0.617 & 0.643 & 0.189 & 0.424 \\
\hline$p$-value & 0.001 & $<0.001$ & 0.356 & 0.031 \\
\hline \multicolumn{5}{|l|}{ Lower vertebra, sagittal } \\
\hline r & 0.567 & 0.616 & 0.228 & 0.440 \\
\hline$p$-value & 0.002 & 0.001 & 0.263 & 0.025 \\
\hline
\end{tabular}

HU : Hounsfield unit, DEXA : dual energy X-ray absorptiometry, BMD : bone mineral density 
0.727; sensitivity, 94.7\%; specificity, 2.9\%) and $22.41 \mathrm{HU}$ at the intraoperative distraction ( $p=0.017$; AUC, 0.722 ; sensitivity, $85.7 \%$; specificity, 57.9\%). Statistical significance was con- firmed, except for lower cortical and sag lower HU values. Using this value, we converted these parameters into a bifurcated variable and assessed the multinomial regression analysis to

Table 2. Demographics of clinical data between the cage decrement group and control group

\begin{tabular}{|c|c|c|c|}
\hline & Cage decrement group $(n=19)$ & Control group $(n=21)$ & $p$-value \\
\hline Age (years) & $55.05 \pm 10.99$ & $49.24 \pm 10.06$ & 0.089 \\
\hline Gender, male : female & $10: 9$ & $11: 10$ & 0.999 \\
\hline BMI & $24.38 \pm 3.89$ & $24.36 \pm 3.42$ & 0.985 \\
\hline DM & 3 & 2 & 0.654 \\
\hline Smoking history & 6 & 5 & 0.727 \\
\hline $\mathrm{EBL}(\mathrm{mL})$ & $88.84 \pm 62.01$ & $76.19 \pm 46.42$ & 0.540 \\
\hline ASA score, 1/2/3 & $10 / 9 / 0$ & $11 / 9 / 1$ & 0.999 \\
\hline Cage depth, $12 \mathrm{~mm} / 14 \mathrm{~mm}$ & $8 / 11$ & $10 / 11$ & 0.761 \\
\hline Cage size, 5/6/7/8 & $2 / 3 / 13 / 3$ & $1 / 6 / 11 / 8$ & 0.375 \\
\hline Operation level & & & 0.683 \\
\hline C3-4 & 2 & 2 & \\
\hline C4-5 & 2 & 5 & \\
\hline C5-6 & 11 & 9 & \\
\hline C6-7 & 4 & 5 & \\
\hline Pseudoarthrosis & 6 & 8 & 0.757 \\
\hline
\end{tabular}

Values are presented as mean \pm standard deviation or number (\%) unless otherwise indicated. BMI : body mass index, DM : diabetes mellitus, EBL : estimated blood loss, ASA : American Society of Anesthesiology

Table 3. Demographics of radiologic data between the cage decrement group and control group

\begin{tabular}{|c|c|c|c|}
\hline & Cage decrement group $(n=19)$ & Control group $(n=21)$ & $p$-value \\
\hline Pre CA & $12.24 \pm 12.91$ & $8.29 \pm 12.28$ & 0.327 \\
\hline Pre SA & $2.95 \pm 6.86$ & $0.48 \pm 5.91$ & 0.228 \\
\hline PreT1 slope & $23.10 \pm 8.05$ & $21.63 \pm 7.13$ & 0.546 \\
\hline Pre SVA & $24.41 \pm 10.46$ & $19.90 \pm 10.07$ & 0.173 \\
\hline Pre TIH & $35.22 \pm 3.74$ & $35.37 \pm 3.87$ & 0.900 \\
\hline \multicolumn{4}{|l|}{ HU value } \\
\hline Upper vertebra, cancellous & $340.2 \pm 100.3$ & $417.7 \pm 100.5$ & 0.019 \\
\hline Upper vertebra, cortical + cancellous & $407.6 \pm 100.5$ & $500.2 \pm 117.3$ & 0.011 \\
\hline Lower vertebra, cancellous & $302.7 \pm 78.3$ & $370.7 \pm 95.1$ & 0.019 \\
\hline Lower vertebra, cortical + cancellous & $361.2 \pm 87.3$ & $440.7 \pm 117.1$ & 0.021 \\
\hline Upper vertebra sagittal & $384.4 \pm 78.4$ & $444.8 \pm 75.4$ & 0.017 \\
\hline Lower vertebra sagittal & $336.4 \pm 80.5$ & $397.9 \pm 105.4$ & 0.047 \\
\hline \multicolumn{4}{|l|}{ Intraoperative change } \\
\hline Variation of CA & $1.28 \pm 7.29$ & $2.02 \pm 7.25$ & 0.750 \\
\hline Variation of SA & $1.83 \pm 5.07$ & $6.93 \pm 5.87$ & 0.006 \\
\hline Variation of $\mathrm{TIH}$ & $1.17 \pm 2.24$ & $3.93 \pm 1.08$ & $<0.001$ \\
\hline Intraoperative distraction & $20.10 \pm 25.56$ & $42.60 \pm 27.76$ & 0.007 \\
\hline
\end{tabular}

Values are presented as mean \pm standard deviation. CA : C2-7 cobb angle, SA : segmental angel, SVA : sagittal vertical axis, TIH : total intervertebral height, HU : Hounsfield unit 
Table 4. Results of ROC curve analysis and multivariate analysis for risk factors

\begin{tabular}{|c|c|c|c|c|c|c|c|c|}
\hline & \multicolumn{5}{|c|}{ ROC curve analysis } & \multicolumn{3}{|c|}{ Multivariate analysis } \\
\hline & AUC & $p$-value & Cut-off & Sensitivity & Specificity & B & $p$-value & $\operatorname{Exp}(B)$ \\
\hline Intraoperative distraction & 0.722 & 0.017 & 22.41 & 0.857 & 0.579 & 2.840 & 0.012 & 17.123 \\
\hline Upper vertebra, cancellous & 0.707 & 0.025 & 425.16 & 0.895 & 0.524 & & & \\
\hline Upper vertebra, cortical + cancellous & 0.727 & 0.014 & 529.84 & 0.947 & 0.429 & 3.427 & 0.017 & 30.771 \\
\hline Lower vertebra, cancellous & 0.692 & 0.038 & 342.81 & 0.737 & 0.619 & & & \\
\hline Lower vertebra, cortical + cancellous & 0.669 & 0.068 & - & - & - & & & \\
\hline Upper vertebra sagittal & 0.707 & 0.025 & 375.89 & 0.526 & 0.857 & & & \\
\hline Upper vertebra sagittal & 0.659 & 0.085 & - & - & - & & & \\
\hline
\end{tabular}

$\mathrm{ROC}$ : receiver operating characteristic, $\mathrm{AUC}$ : area under the curve

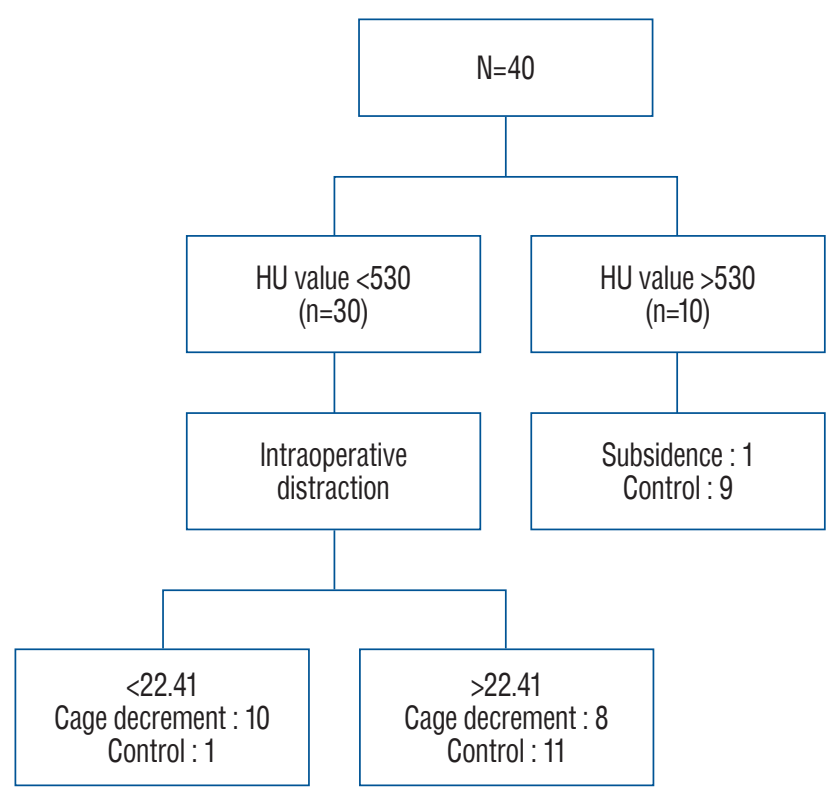

Fig. 4. The flow for each of the two risk factors of the cage decrement. $\mathrm{HU}$ : Hounsfield unit.

evaluate the risk factors of postoperative decrement of ACDF. Finally, we confirmed that intraoperative distraction and $\mathrm{HU}$ value of the upper vertebral body were independent of postoperative subsidence. The flow chart for each of the two risk factors has been presented in Fig. 4 .

If the patient had two risk factors, $90 \%$ of cage decrement after ACDF was predicted. The patient data was analyzed by applying the previous criteria defined by more than $3 \mathrm{~mm}$ cage subsidence after ACDF surgery and compared the composition of each group (Tables 5 and 6). Cage subsidence was present in 18 patients. In nine of 11 patients with two risk factors, cage subsidence occurred. A probability of $80 \%$ was able to predict the cage subsidence. The criteria for cage decrement
Table 5. Cross table according to risk scores

\begin{tabular}{lccc}
\hline Risk score & Sample size & Cage decrement group & Control \\
\hline 0 & 7 & $0(0.0)$ & $7(100.0)$ \\
1 & 22 & $9(40.9)$ & $13(59.1)$ \\
2 & 11 & $10(90.9)$ & $1(9.1)$ \\
Total & 40 & 19 & 21 \\
\hline
\end{tabular}

Values are presented as number (\%). Cross table between cage decrement group $(n=19)$ and control group $(n=21)$

Table 6. Cross table according to risk scores

\begin{tabular}{lccc}
\hline Risk score & Sample size & $\begin{array}{c}\text { Cage subsidence } \\
\text { group }\end{array}$ & $\begin{array}{c}\text { Non cage } \\
\text { subsidence group }\end{array}$ \\
\hline 0 & 7 & $1(14.3)$ & $6(85.7)$ \\
1 & 22 & $8(36.4)$ & $14(63.6)$ \\
\hline 2 & 11 & $9(81.8)$ & $2(18.22)$ \\
\hline Total & 40 & 18 & 22 \\
\hline
\end{tabular}

Values are presented as number (\%). Cross table between cage subsidence group ( $n=18)$ and non-cage subsidence group ( $n=22)$

did not differ significantly in patient composition compared to those with $3 \mathrm{~mm}$ cage subsidence.

A graph showing the variation in $\mathrm{TIH}$ according to the flow of time for each group involving risk factors has been presented in Fig. 5. A gentle slope was observed in patients with an $\mathrm{HU}$ value $>530$ compared to those with an $\mathrm{HU}$ value $<530$. Patients with adequate intraoperative distraction had no difference in the slope of the graph compared to the other patients. However, the final TIH was not reduced when compared to the preoperative TIH due to increased height. 

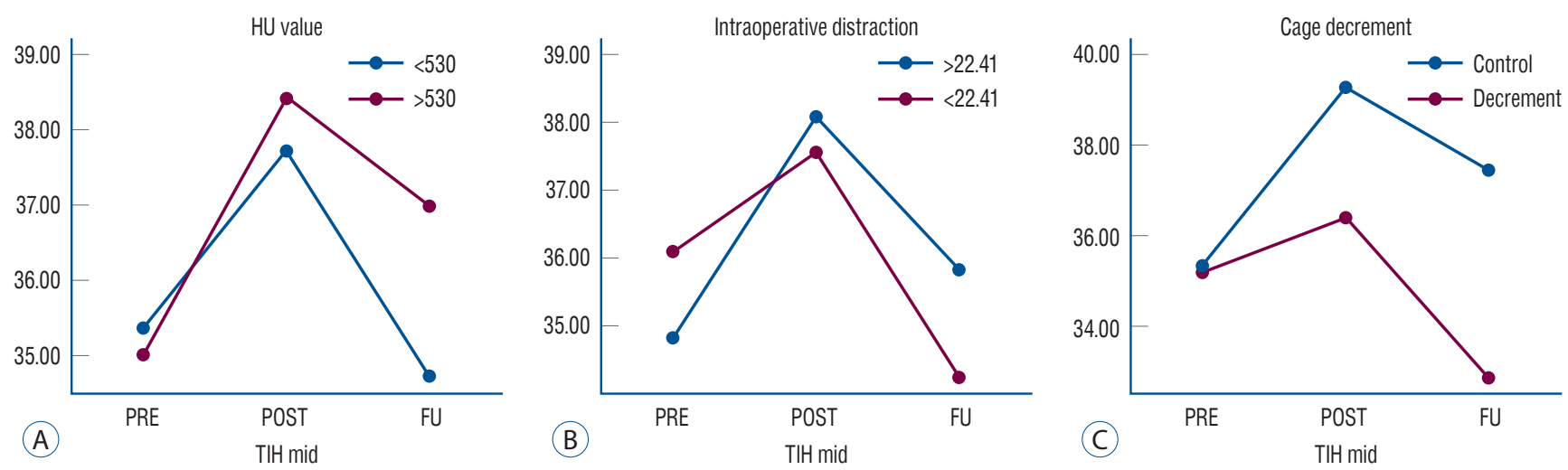

Fig. 5. Change of the mean value of total intervertebral height according to the risk factors from preoperative to follow up period. A : Risk factor : HU value $<530$ (upper vertebral, cortical + cancellous). B : Risk factor : intraoperative distraction $<22.41$. C : Change of average total intervertebral height at cage decrement group and control group. $\mathrm{HU}$ : Hounsfield unit, $\mathrm{FU}$ : follow up, $\mathrm{TIH}$ : total intevertebral height.
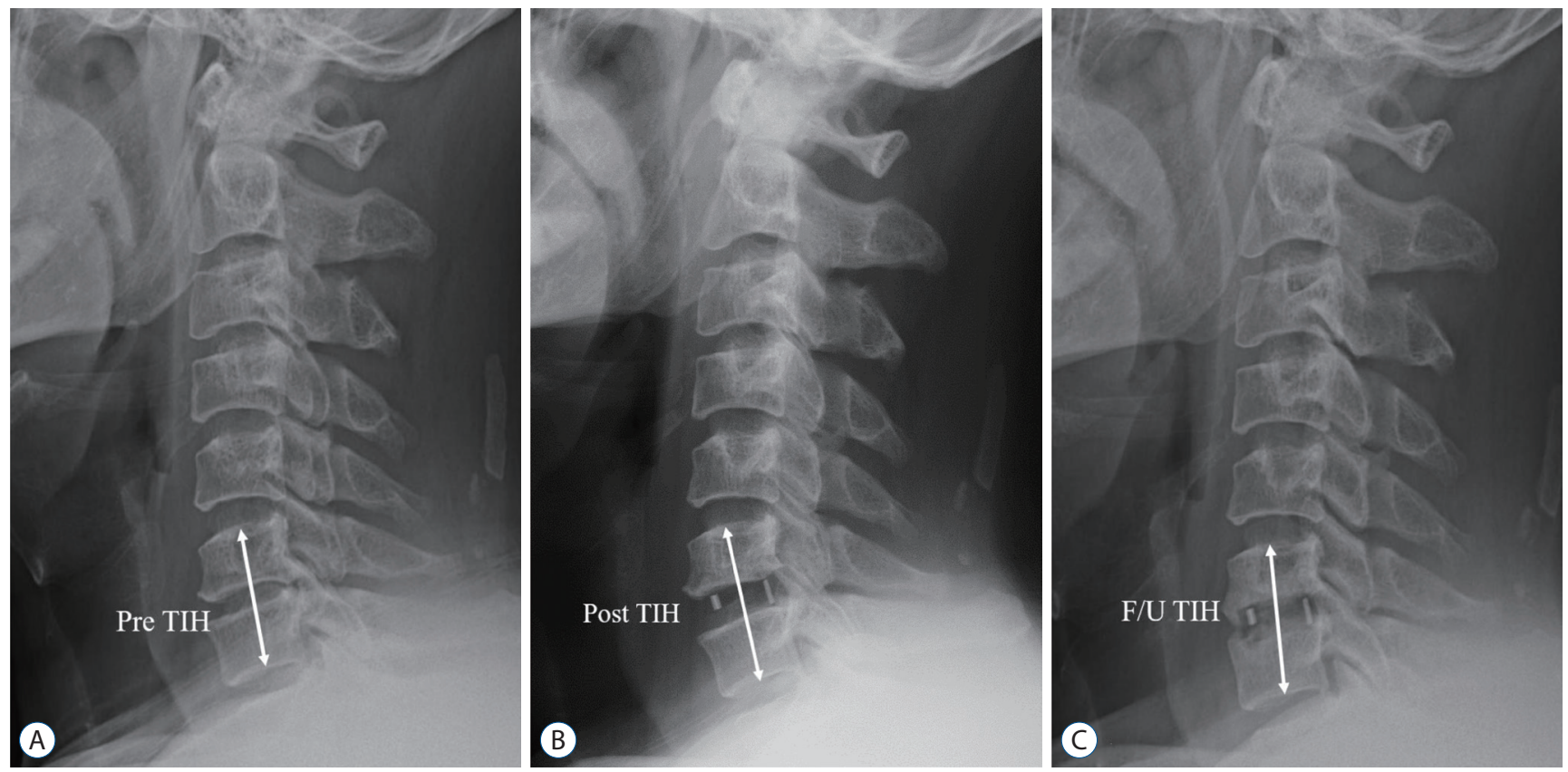

Fig. 6. A 59-year old man patient presented with tingling sensation of both arm, C6/7 ACDF. A : Preoperative TIH : $35.34 \mathrm{~mm}$. B : Postoperative TIH : 39.21 $\mathrm{mm}$. C : F/U TIH : $35.44 \mathrm{~mm}$. This case belonged to cage subsidence group, but it did not belong to cage decrement group (pre $\mathrm{TIH}: 35.34 \mathrm{~mm}<\mathrm{F} / \mathrm{U} \mathrm{TIH}$ : 35.44). $\mathrm{TIH}$ : total intevertebral height, F/U : follow up.

\section{DISCUSSION}

ACDF using stand-alone PEEK is the most advantageous surgical strategy for cervical degenerative disease. With this procedure, we prevented the problem related to anterior cervical plating, including screw loosening, screw migration, and dysphagia due to soft tissue swelling ${ }^{21)}$. However, according to the literature, there are many postoperative complications. Of various postoperative complications, graft subsidence be- longed to major complications. Although there is some controversy over the criteria for postoperative subsidence, the incidence of graft subsidence after ACDF with stand-alone PEEK cage is reported to be $8.1-44.7 \%^{2}$. Although studies to identify the risk factors related to graft subsidence have been conducted, a consensus has not been achieved. As mentioned previously, there are several problems with the study of cage subsidence. Therefore, we introduce a new concept called cage decrement, focusing on whether the preoperative vertebral 
height is maintained. Concept of cage subsidence concentrated on the results that graft penetrated on the vertebral endplate, making it difficult to confirm whether preoperative height of neural foramen and vertebral height are maintained. In concept of cage decrement, the use of high-height cage acted as an independent variable contributing the increase of TIH. However, in concept of cage subsidence, the use of highheight cage acted as a dependent variable that exacerbates the cage subsidence. In Fig. 6, we introduced illustrated case. This case belonged to cage subsidence group, but it did not belong to cage decrement group. Although graft subsidence has been occurred, with use of the sufficiently high height cage, TIH and SA has been preserved.

As shown in Tables 5 and 6, classification by the new cage decrement criteria did not differ significantly from the previous classification based on the $3 \mathrm{~mm}$ cage subsidence criteria. However, when classified as per the $3 \mathrm{~mm}$ criterion, the multiple regression analysis did not produce statistically significant risk factors.

The identified risk factors in our study were the HU values and intraoperative distraction. The method of measuring the bone quality of the surgical lesion by checking the HU value in the operative lesion unit has been used in several studies. Schreiber et al. ${ }^{15)}$ introduced the HU measurement of each vertebral body using the standard PACS software. Unlike the lumbar spine, studies using the HU value of the cervical bone are lacking. Recent studies have evaluated the use of HU units of the commercial bone. Wang et al. ${ }^{18)}$ reported that lower preoperative CT HU values are associated with cage subsidence in single-level ACDF. Their study was similar to ours however, the following differences were noted. First, our research targeted patients who performed single-level ACDF without anterior plating. Moreover, the methods for measuring HU values were different. Second, we analyzed additional risk factors to forecast graft subsidence.

There are many studies on the association between the low HU values and mechanical failure in lumbar fusion surgery $^{13,14)}$. However, efforts to use HU values have been relatively limited in ACDF surgery and DEXA is not universally obtained by all spine surgeons before $\mathrm{ACDF}^{5)}$. Our results showed that the cause of cage decrement depends on the bone quality and the HU value of conventional CT scans can reflect cervical bone quality. HU values also confirmed high consistency with lumbar BMD rather than that in femur heads and identified the usefulness of preoperative DEXA examination in cervical spine operation.

There is no consensus on how to measure the HU value of the vertebral body. Schneider et al. ${ }^{15)}$ first introduced the widely used measurement of HU values. The HU values of the cancellous bone of the vertebral body were measured at three axial image heights parallel to the endplates. However, the sagittal planes of the vertebral body were not measured. Lee et al.'s study ${ }^{11)}$ measured the vertebral body using sagittal and axial planes. Considering the characteristics of the cervical bone in which sclerotic changes of the vertebral body are frequently observed, we measured the vertebral body in three ways, including the cortical bone of the cervical bone. Although the most significant areas was the upper cortical vertebra including the cortical bone, statistical significance was also confirmed in other in other ways. We have a question why measuring the HU value in upper vertebral is the most valid site. According to the prior study about the trabecular bone density of cervical and lumbar vertebra ${ }^{22)}$, BMD value of high cervical vertebra is higher than BMD value of low cervical vertebra in normal human body. It can be assumed that cephalic cervical vertebra might reflect the quality of the entire bone better than caudal cervical vertebra, however additional study will be needed.

Intraoperative distraction has also been regarded as a cause of graft subsidence in some previous study ${ }^{7,17,21)}$. In general, excessive intraoperative distraction caused a high static compressive force on the intervertebral endplate surface and potentially accelerated cage subsidence. However, it has a problem that PEEK cage with higher height should not be used. There was no objective parameters to use for determining the appropriate size during operation ${ }^{7)}$ and it is not easy to confirm whether the distractive force is appropriate or not. Adequate compressive force to endplate could be prevented the cage migration. And since our cases did not performed the anterior plate anchoring, we are free from the instrument failure such as screw back out or plate loosening caused by pull out force secondary to cage subsidence. In our study, we could confirm that cage decrement was most affected by the difference in HU values, that is, the cervical bone quality. Patients with relatively elevated disc height could eventually secure the disc space height and maintain lordosis of the SA. Intraoperative distraction is a factor that involves the surgeon's discretion, and selecting a high-sized well-fitting graft could achieve 
a good radiologic outcome during ACDF surgery. However, excessive intraoperative distraction may reduce load transmission in the posterior column, worsening postoperative cervicalgia $^{3)}$.

We conducted the retrospective study after defining a cage decrement based on POD 6 months. According to the prior study of cage subsidence after ACDF, the degree of cage subsidence was steepest during the first month after operation ${ }^{1,8}$. Afterward, the degree of cage subsidence gradually had slowed down until 6 months and the operative lesion has been stabilized after formation of fibrous union. Although there may be some exceptional cases, we set the criterion as POD 6 months in the study. Progression of cage subsidence was associated with degree of segmental instability (micro-motion) ${ }^{9,19)}$ and bony bridge formation ${ }^{21)}$. Unfortunately, it is not feasible to perform CT examination on all cases. So, we measured the inter-spinous distance on flexion-extension lateral radiography and total pseudoarthrosis rates was $28 \%$. This result was thought to be inferior compared to previously published result $^{18,21}$. We thought that the main reason is absence anterior plate anchoring and excessive endplate damage during the end plate preparation.

This study has some limitations. First, it was a small retrospective study, and the follow-up period to confirm postoperative radiologic changes was short. And the age range of enrolled patients was diverse. So these aspects may reduce the confidence of the results. Second, our study introduced a new concept called cage decrement to accurately determine the degree of graft subsidence after ACDF. However, we have not evaluated comparative studies on whether these new concepts are clinically useful due to the limitation of a small number of patients. Third, we did not conduct a DEXA examination in all patients; it was performed in 27 out of 40 patients, which is a limitation in determining the usefulness of the DEXA examination.

\section{CONCLUSION}

Insufficient intraoperative distraction and low $\mathrm{HU}$ value showed a strong relationship with postoperative intervertebral height reduction following single stand-alone PEEK cage ACDF.

\section{CONFLICTS OF INTEREST}

No potential conflict of interest relevant to this article was reported.

\section{INFORMED CONSENT}

This type of study does not require informed consent.

\section{AUTHOR CONTRIBUTIONS}

\author{
Conceptualization : JSL, DWS \\ Data curation : SSK \\ Formal analysis : DWS \\ Funding acquisition : JSL \\ Methodology : DWS, SWL \\ Project administration : SSK \\ Visualization: SHL, JBW, YHK \\ Writing - original draft : JSL \\ Writing - review \& editing : JSL, DWS, SHL, SWL, GSS
}

\section{ORCID}

$\begin{array}{ll}\text { Jun Seok Lee } & \text { https://orcid.org/0000-0003-2488-6953 } \\ \text { Dong Wuk Son } & \text { https://orcid.org/0000-0002-9154-1923 } \\ \text { Su Hun Lee } & \text { https://orcid.org/0000-0001-8952-5556 } \\ \text { Sung Soon Ki } & \text { https://orcid.org/0000-0001-7138-9621 } \\ \text { Sang Weon Lee } & \text { https://orcid.org/0000-0002-3199-7072 } \\ \text { Geun Sung Song } & \text { https://orcid.org/0000-0001-8029-9011 } \\ \text { Joon Bum Woo } & \text { https://orcid.org/0000-0003-1803-8763 } \\ \text { Young Ha Kim } & \text { https://orcid.org/0000-0003-0477-3169 }\end{array}$

\section{- Acknowledgements}

This study was supported by Research Institute for Convergence of Biomedical Science and Technology (30-2021-000), Pusan National University Yangsan Hospital. 


\section{References}

1. Barsa P, Suchomel P : Factors affecting sagittal malalignment due to cage subsidence in standalone cage assisted anterior cervical fusion. Eur Spine J 16 : 1395-1400, 2007

2. Brenke C, Dostal M, Scharf J, Weiß C, Schmieder K, Barth M : Influence of cervical bone mineral density on cage subsidence in patients following stand-alone anterior cervical discectomy and fusion. Eur Spine J 24 : 2832-2840, 2015

3. Choi MK, Kim SB, Lee JH : Rare intractable cervicalgia related to exaggerated disc height distraction : report of two cases and literature review. J Korean Neurosurg Soc 61 : 530-536, 2018

4. Coe JD, Warden KE, Herzig MA, McAfee PC : Influence of bone mineral density on the fixation of thoracolumbar implants. A comparative study of transpedicular screws, laminar hooks, and spinous process wires. Spine (Phila Pa 1976) 15 : 902-907, 1990

5. Dipaola CP, Bible JE, Biswas D, Dipaola M, Grauer JN, Rechtine GR : Survey of spine surgeons on attitudes regarding osteoporosis and osteomalacia screening and treatment for fractures, fusion surgery, and pseudoarthrosis. Spine J 9 : 537-544, 2009

6. Jergas M, Breitenseher M, Glüer CC, Black D, Lang P, Grampp S, et al. : Which vertebrae should be assessed using lateral dual-energy $X$-ray absorptiometry of the lumbar spine. Osteoporos Int 5 : 196-204, 1995

7. Kao TH, Wu CH, Chou YC, Chen HT, Chen WH, Tsou HK : Risk factors for subsidence in anterior cervical fusion with stand-alone polyetheretherketone (PEEK) cages: a review of 82 cases and 182 levels. Arch Orthop Trauma Surg 134 : 1343-1351, 2014

8. Kast E, Derakhshani S, Bothmann M, Oberle J : Subsidence after anterior cervical inter-body fusion. A randomized prospective clinical trial. Neurosurg Rev 32 : 207-214; discussion 214, 2009

9. Kettler A, Wilke HJ, Claes L : Effects of neck movements on stability and subsidence in cervical interbody fusion: an in vitro study. J Neurosurg 94(1 Suppl) : 97-107, 2001

10. Kulkarni $A G$, Hee HT, Wong HK : Solis cage (PEEK) for anterior cervical fusion: preliminary radiological results with emphasis on fusion and subsidence. Spine J 7 : 205-209, 2007

11. Lee SJ, Binkley N, Lubner MG, Bruce RJ, Ziemlewicz TJ, Pickhardt PJ : Opportunistic screening for osteoporosis using the sagittal reconstruction from routine abdominal CT for combined assessment of vertebral fractures and density. Osteoporos Int 27 : 1131-1136, 2016

12. Lochmüller EM, Bürklein $D$, Kuhn V, Glaser $C$, Müller $R$, Glüer $C C$, et al. : Mechanical strength of the thoracolumbar spine in the elderly: prediction from in situ dual-energy $\mathrm{X}$-ray absorptiometry, quantitative computed tomography (QCT), upper and lower limb peripheral QCT, and quantitative ultrasound. Bone 31 : 77-84, 2002

13. Mi J, Li K, Zhao X, Zhao CQ, Li H, Zhao J : Vertebral body Hounsfield units are associated with cage subsidence after transforaminal lumbar interbody fusion with unilateral pedicle screw fixation. Clin Spine Surg 30 : E1130-E1136, 2017

14. Sakai Y, Takenaka S, Matsuo Y, Fujiwara H, Honda H, Makino T, et al. : Hounsfield unit of screw trajectory as a predictor of pedicle screw loosening after single level lumbar interbody fusion. J Orthop Sci 23 : 734738, 2018

15. Schreiber JJ, Anderson PA, Rosas HG, Buchholz AL, Au AG : Hounsfield units for assessing bone mineral density and strength: a tool for osteoporosis management. J Bone Joint Surg Am 93 : 1057-1063, 2011

16. Singh K, Vaccaro AR, Kim J, Lorenz EP, Lim TH, An HS : Biomechanical comparison of cervical spine reconstructive techniques after a multilevel corpectomy of the cervical spine. Spine (Phila Pa 1976) 28 : 23522358; discussion 2358, 2003

17. Truumees E, Demetropoulos CK, Yang KH, Herkowitz HN : Effects of disc height and distractive forces on graft compression in an anterior cervical discectomy model. Spine (Phila Pa 1976) 27 : 2441-2445, 2002

18. Wang M, Mummaneni PV, Xi Z, Chang CC, Rivera J, Guinn J, et al. : Lower Hounsfield units on CT are associated with cage subsidence after anterior cervical discectomy and fusion. J Neurosurg Spine 33 : 425432, 2020

19. Wu SH, Li Y, Zhang YQ, Li XK, Yuan CF, Hao YL, et al. : Porous titanium- 6 aluminum- 4 vanadium cage has better osseointegration and less micromotion than a poly-ether-ether-ketone cage in sheep vertebral fusion. Artif Organs 37 : E191-E201, 2013

20. Wu WJ, Jiang LS, Liang Y, Dai LY : Cage subsidence does not, but cervical lordosis improvement does affect the long-term results of anterior cervical fusion with stand-alone cage for degenerative cervical disc disease: a retrospective study. Eur Spine J 21 : 1374-1382, 2012

21. Yang JJ, Yu CH, Chang BS, Yeom JS, Lee JH, Lee CK : Subsidence and nonunion after anterior cervical interbody fusion using a stand-alone polyetheretherketone (PEEK) cage. Clin Orthop Surg 3 : 16-23, 2011

22. Yoganandan N, Pintar FA, Stemper BD, Baisden JL, Aktay R, Shender $B S$, et al. : Trabecular bone density of male human cervical and lumbar vertebrae. Bone 39 : 336-344, 2006 